\title{
Ultrastructural characterization of the embryonic egg and the L1 larva of Trichuris muris
}

Ludmila Rocha Lima ${ }^{1}$ and Eduardo José Lopes Torres ${ }^{1} *$

1. Laboratório de Helmintologia Romero Lascasa Porto (LHRLP), FCM-UERJ, RJ, Brasil. *lopestorresej@gmail.com

Soil-transmitted helminthiases are neglected diseases developed by nematodes that depend on the soil for their complete development; having an impact on human and veterinary medicine[1]. The species Trichuris muris is used as an experimental model for trichuriasis, the life cycle begins with the ingestion of embryonated eggs by the host[2]. The aim of this work is the ultrastructural characterization of the embryonated egg and the L1 larva of Trichuris muris fixed by high-pressure freezing followed by freeze-substitution and analyzed by electron microscopy. Chemically fixed samples were stained with calcein and DAPI for fluorescence microscopy,confocal and super-resolution microscopy resolution experiments. The results obtained by light microscopy (LM) (Fig. 1A) showed the three layers forming the trilaminar egg shell (vitelline, chitin and lipid layers). The larva esophagus (Es), germ cells (GC) concentrated in the posterior region of the larva was also observed and cell concentration that will possibly give rise to the structure known as the bacillary band $(\mathrm{Bb})$. Each pole has a polar plug (PP). Using the confocal microscopy and super-resolution microscopy (Fig. 1B) was possible to present the high affinity of the calcein with the eggshell and the polar plug. While through the DAPI it was possible to stain each germ cell of the larva. Based in the image series obtained by confocal experiments, we performed the reconstruction and modeling of germ cells, enabling the quantification of $151 \pm 28$ (130-182) cells in each larva (Fig. 1C). In addition, was possible to visualize two cells in division (telophase stage) (Fig. 1D, inset). Using TEM was possible to describe the polar plug formed by the of cuticulin layer modified (Fig. 2A), presenting a better structural preservation. The eggshell present 14 layers: one vitelline layer, cuticulin layer with 12 divisions and one lipid layer. Between the eggshell and the larva, the extra embryonic matrix and, more internally, the permeability membrane were observed (Fig. 2C). The stylet structure was identified in anterior end of the larva and the cuticle striations were observed too (Fig. 2D). We conclude that the cryofixation and the use of the different microscopy techniques were very important to characterize in detail of the morphology and morphometry of the different eggshell structures, including the characterization of the L1 larva, such as the quantification/distribution of germ cells.
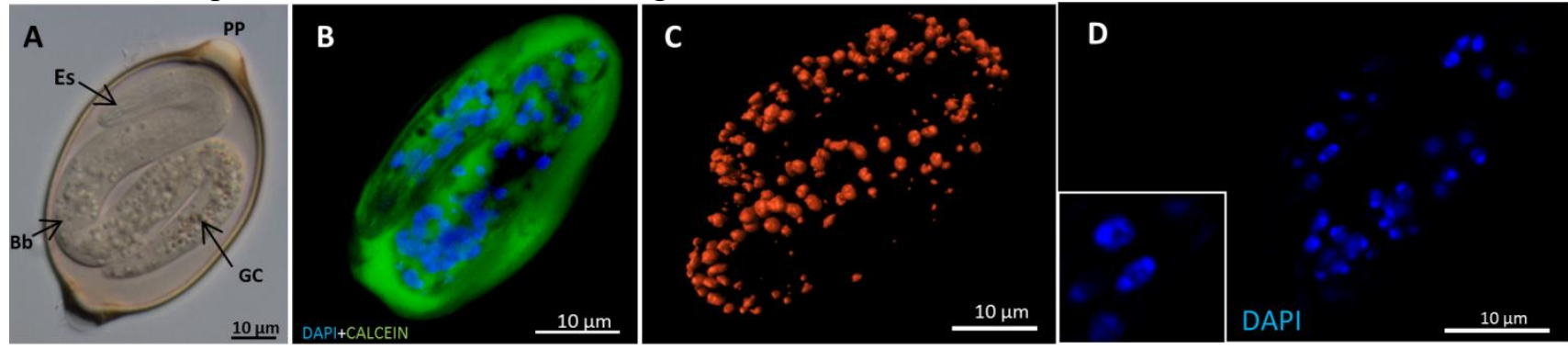

Figure 1 Light microscopy of the T. muris egg structures: (A) Bright field showing the polar plug (PP), larva L1 with esophagus (Es) and germ cells (GC) associated with the formation of the bacillary band (Bb) and digestive system. (B) Confocal image showing larva germ cells (blue) and egg content (green). Fig (C) Threedimensional modeling of the L1 stage germ cells. (D) Fluorescence image of the germ cells, showing the cell division. Inset: detail of two cells in telophase stage. 


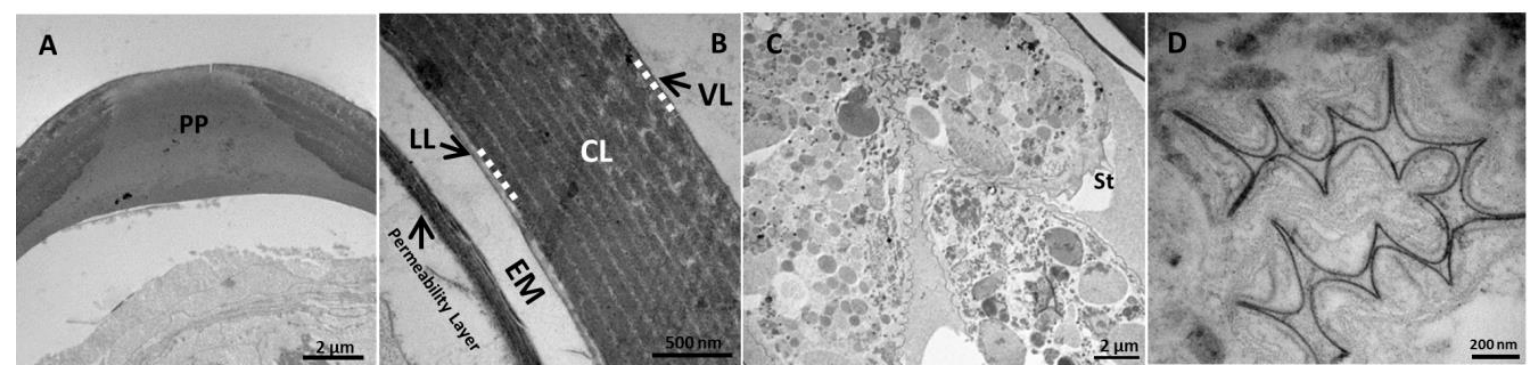

Figure 2 Transmission electron microscopy of the T. muris egg structures: (A) Detail of the polar plug (PP). (B) Different layers of the eggshell (VL: Vitelline, CL: Cuticulin, LL: Lipid), extraembryonic membrane (EM) and permeability layer (Asterisk). (C) L1 larva with the stylet (St) in the anterior end and the cuticular striation of the body. (D) Detail of the cuticular striations in the mid-region of the nematode body.

References:

[1] WHO, W.H.O. Helmintiasis transmitidas por el suelo , 2019.

[2] PRESTON, C. M.; JENKINS, T.. Parasitology, v. 89, (1984) 263. 